\title{
A Study of the Role of Organized Structures in Jet Noise Generation
}

\author{
R. Reba* and S. Narayanan ${ }^{\dagger}$ \\ United Technologies Research Center, E Hartford, CT \\ T. Colonius $\AA^{\ddagger}$ and M. J. Dunlop§ \\ California Institute of Technology, Pasadena, CA
}

\begin{abstract}
We present analytical, experimental and computational studies aimed at understanding the role of large-scale, organized structures in noise generation from high-speed, compressible jets. Two-point near-field pressure data from experiments are analyzed and used to identify parameters in a wave-packet based model for noise generation from organized, large-scale structures. The statistical spectrum of far field pressure is expressed in terms of two-point space-time correlations of the near-field pressure on a surface enclosing the jet. The surface is assumed to be sufficiently near the turbulent region to be dominated by non-propagating hydrodynamic disturbances, yet sufficiently far such that linear behavior can be assumed in extending the near-field pressure to the far field. Validity of such assumptions is investigated by interrogating the jet DNS database of Freund [J. Fluid Mech. 438:277-305 2001]. The DNS data analysis is also used to investigate the impact of limited spatial resolution in the experiments. The analytical model is used to study far field noise generation from organized structures. Results show that, for sufficiently short structure lifetime, aft angle far field pressure spectra tend to exhibit frequency scaling with Helmholtz number, rather than Strouhal number.
\end{abstract}

\section{Introduction}

Significant effort has been expended in understanding the sources of jet noise generation over the past fifty years dating back to the seminal work of Lighthill, ${ }^{1}$ who proposed an acoustic analogy framework to describe sound generation from turbulence. Computations are only recently demonstrating accurate capture of the relevant sources and their noise signature. $^{2}$ It now seems to be generally accepted that high-speed jet noise generation is comprised of two mechanisms with distinct directivities and spectral content originating from fine-scale turbulence and large-scale, organized aspects of turbulence. The former mechanism has received significant attention resulting in the development of semi-empirical models. ${ }^{3}$ On the other hand, very little in the way of analytical modeling or prediction approaches for realistic exhausts exist concerning the relationship between large-scale, coherent motion and the low frequency sound generation in the downstream angles. Largeeddy simulations ${ }^{4}$ for jet noise are only now becoming feasible at sufficiently high Reynolds numbers, and reduced-order models ${ }^{5,6}$ of noise generation from organized structures are in their infancy.

\footnotetext{
*Research Engineer, Acoustics Group

${ }^{\dagger}$ Associate Fellow, Systems Department, Member AIAA

$\ddagger$ Associate Professor of Mechanical Engineering, Member AIAA

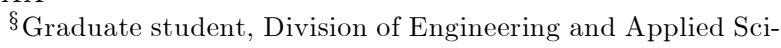
ence
}

The understanding of jet turbulence itself was advanced by the finding of orderly, large-scale coherent structures, which have been the focus of several explorations of turbulence generation and evolution. ${ }^{7}$ The preferential amplification of disturbances in certain frequency bands leads to the formation, interaction and subsequent decay of structures in the jet. One might therefore consider describing the acoustic behavior of the jet based on the growth and decay of a coherent structure. Such a description closely parallels the association of sound generation with linear instability waves pursued by Crighton and co-workers ${ }^{8,9}$ for very low Mach number flows. They focused on the superdirective nature of the noise radiation.and its dependence on the envelope shape (inferred from Laufer and Yen ${ }^{10}$ measurements of near-field pressure in a jet) and size (relative to the acoustic wavelength). On the other hand, Morris and Tam, ${ }^{11}$ have computed far field sound from linear instability waves for high supersonic jet flows, where a supersonic convective wave speed (relative to the ambient sound speed) enables efficient coupling with the acoustic field. Such analytical and computational descriptions have been less successful in high subsonic jets, relevant to commercial aircraft noise applications, where only a small portion of the frequency-wavenumber distribution of the relevant scales of motion extend to the radiating supersonic region.

Evidently, the connection between large-scale structures in a jet flow field and the far field noise generation 
is a subject of ongoing research. Particularly lacking is a framework to analyze the sound generation process from organized structures to better understand the sensitivity of the acoustic field to changes in the flow structure evolution in a parametric fashion. Implications of such an understanding on the development of innovative and effective jet noise reduction concepts are obvious.

From an experimental standpoint, several approaches to inferring the sound sources have been proposed that would avoid retarded time integration of the Lighthill source. Some of the earliest measurements of pressure fluctuations due to coherent structures in the jet near field and their connection with noise radiation were reported by Mollo-Christensen. ${ }^{12}$ Zaman $^{13}$ explored the near and far field sound of a modest Mach number and Reynolds number subsonic jet, attempting to describe noise source locations in terms of turbulence characteristics and to infer their relationship with coherent structures in the jet, although no direct correlations were attempted.

There have been several measurements of pointwise quantities such as density, velocity and vorticity fluctuations simultaneously with far field noise measurements. Researchers have used these to establish a cause-and-effect relationship between turbulence characteristics and far field noise, dating back to the works of Siddon, ${ }^{14}$ Seiner and Reethof, ${ }^{15}$ and Armstrong et al. ${ }^{16}$ In an attempt to understand the effect of coherent structures on jet noise and motivated by the measurements of pressure correlations in the (such as by Fuchs ${ }^{17}$ ), Michalke ${ }^{18}$ analytically investigated the effect of the spatial extent of the source coherence on noise generation. The results, based on the solution of the Lighthill equation, reveal that for jets with convection Mach number near unity, larger and 'focused' noise radiation in the aft angles occurs with increasing source coherence (relative to a length scale based on the source volume). More recently, non-intrusive means to attack the causality approach to noise generation have been pursued, ${ }^{19}$ but continued to rely on point measurements. Such measurements capture local turbulence characteristics, but are less effective in describing flow motions over spatially extended regions. The correlation of local flow quantities with the far field sound is found to be small and limited to low frequencies for which the sources are coherent over a large flow region.

Measurements of non-local (or "global") statistics such as the space-time correlation from velocity measurements is only now becoming feasible with the advent of advanced diagnostics such as particle image velocimetry. ${ }^{20}$ Recent studies, ${ }^{21,22}$ have also attempted to relate (albeit qualitatively) the development and interaction of large-scale structures within the jet shear layers to the far field sound generation at low frequencies and in the aft angles. The origin of sound waves

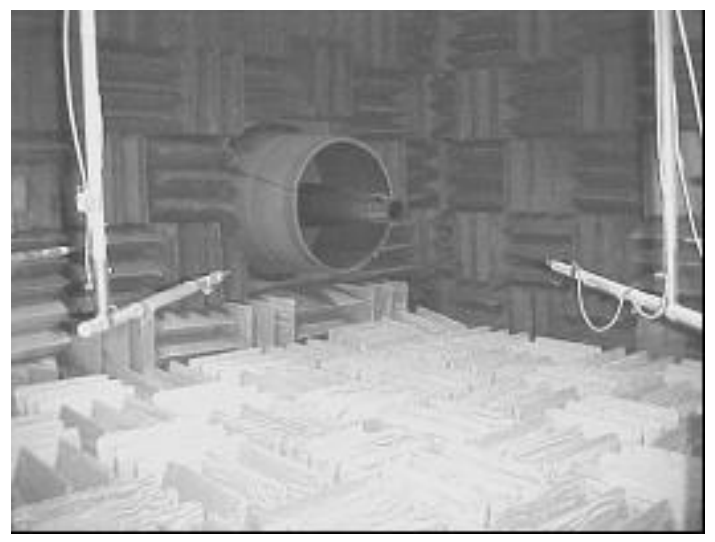

Fig. 1 UTRC Acoustic Research Tunnel.

were inferred from the phase lag between individual microphones of a small array of far field microphones. Coupled with simultaneous visualizations of the instantaneous flow field, such studies provided insights into the evolution of flow structures and the peak noise generation. However, a more direct approach to verifying the validity and extent of contribution of the flow structures to the far-field sound is still lacking. In particular, the aforementioned measurements have not been used to project sound levels in the far field. The present study aims to fill this gap by quantifying the spectral contribution of jet flow structures to the far field sound using semi-empirical analysis.

\section{Experimental Facility and Method}

Figure 1 shows a photograph of the UTRC Acoustic Research Tunnel (ART) in which the experiments were performed. The ART facility is described in detail elsewhere. $^{23}$ The experiments were conducted in a 3.239 " axisymmetric, single stream circular jet housed in the large anechoic chamber with capability to simulate flight effects; details concerning the aero-acoustic characterization of this jet can be found in Simonich et al. ${ }^{23}$ and Narayanan et al. ${ }^{23}$ The tunnel is an open circuit design, which uses a $1500 \mathrm{hp}$ fan to draw outside air through the open jet test section. The test section is a $15 \mathrm{ft} \times 18 \mathrm{ft} \times 22 \mathrm{ft}$ anechoic chamber, designed to provide an anechoic environment down to $200 \mathrm{~Hz}$. A propane combustor supplies heated air up to $1100^{\circ} \mathrm{F}$. Measurements for two jet Mach numbers $M_{j}=0.6,0.9$ are presented here for hot $\left(T_{j}=1000^{\circ} \mathrm{F}\right)$ jet conditions. The present studies were conducted for static jet conditions only, i.e. with no ambient flow, although a small amount of ambient flow is naturally entrained in the open-loop facility (having $M \leq 0.05$ ).

Acoustic data were measured with a set of $3 \mathrm{mi}-$ crophones in the near field and a far-field array of 8 microphones. All microphones used for this study were $1 / 4$ in. B\&K 4135 type. Grids were retained on the microphones for the near field measurements where frequencies only below $10 \mathrm{kHz}$ were considered. 

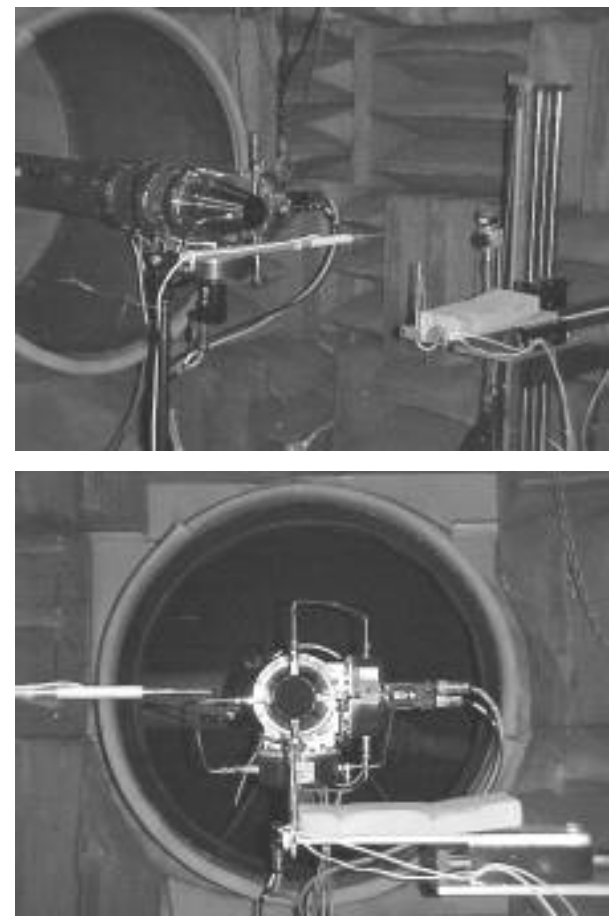

Fig. 2 Near field microphone set up photograph, displaying the single stream jet, one fixed microphone (oriented horizontally) at $x / D_{j}=6$ and a microphone (oriented vertically) mounted on a traverse. A third fixed microphone (not seen here) was placed at $x / D_{j}=2$ oriented at $45^{\circ}$ to the two displayed microphones. Also shown are four tubes from an actuator used to sinusoidally excite the jet shear layer; results for this will be reported elsewhere.

The amplitude response for each microphone was calibrated using a piston phone at $1 \mathrm{kHz}$. The far field microphones were calibrated to $114 \mathrm{~dB}$ for a $500 \mathrm{mVrms}$ piston phone signal, whereas due to the high sound pressure levels encountered by the near field microphones those probes were calibrated to $124 \mathrm{~dB}$ for a $75 \mathrm{mVrms}$ signal. One of the near field microphones was placed on a remotely controlled traverse, used to move the probe and enable placement at several radial and streamwise locations. Three microphones were placed just outside the jet shear layer to enable near field dynamic pressure measurements. Figure 2 shows a couple of views for placement of 2 microphones; a third microphone was also used for these measurements but not shown here. The three microphones were displaced circumferentially (by $45^{\circ}$ each) to minimize wake interference effects (due to the slow ambient flow). Background data with no jet flow and the small ambient tunnel flow were acquired to ascertain that the dynamic pressure levels recorded by the near field microphones were at least $35-40 \mathrm{~dB}$ below the levels encountered when the hot jet was operated.

Two of the near field microphones were placed at fixed locations of $x / D_{j}=2,6$ downstream of jet noz-

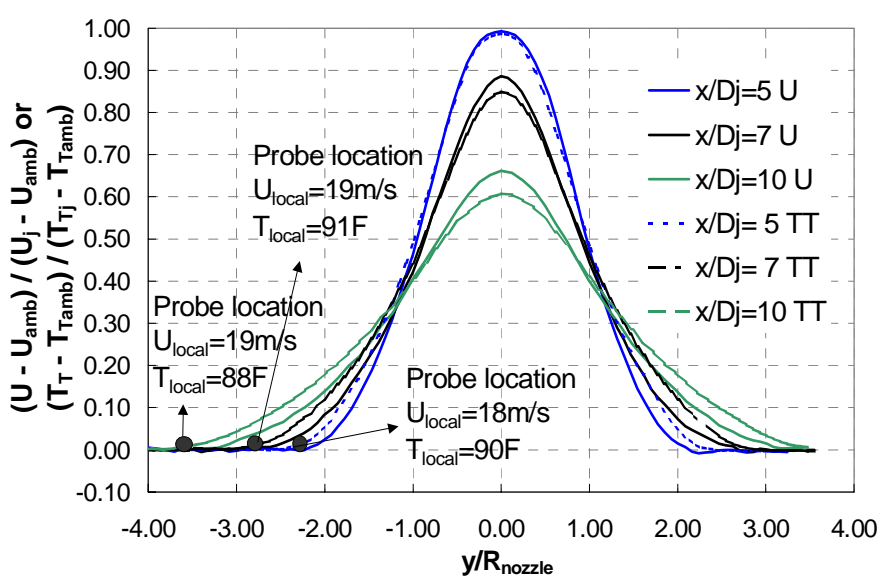

Fig. 3 Normalzied velocity ( $U$, see solid lines) and total temperature $\left(T_{t}\right.$, see dashed lines) profiles for $M_{j}=0.9, T_{j}=1000 \mathrm{~F}$ (from Simonich et al. ${ }^{23}$ measurements), showing location of traversing probe relative to the jet shear layer edge for $5<x / D_{j}<10$.

zle exit plane. The end of the potential core for the $M_{j}=0.6,0.9$ conditions is at approximately $x / D_{j}=3$ and $x / D_{j}=4$, respectively. ${ }^{23}$ The microphone radial locations were chosen to be 0.5-1 in. away from the outer edge of the jet shear layer, to enable detection of near field hydrodynamic pressure fluctuations. For the heated jet flows reported here, the outer shear layer edge was also detected using a thermocouple mounted on the microphone. The microphone was placed in a region where the local temperatures would not exceed 90F. The third traversing microphone was moved along a $7^{\circ}$ inclined line parallel to the outer edge of the time-averaged jet shear layer. Some microphone radial locations are illustrated in Figure 3. As will be evident in later discussions, such placement of the microphones enabled measurements of the hydrodynamic pressure fluctuations for a limited range of frequencies, namely $\mathrm{f}<5 \mathrm{kHz}$. For higher frequencies, it was clear that only acoustic pressure field was being sensed. The radial placement was chosen to maximize the range of frequencies over which the signature of hydrodynamic pressure fluctuations can be captured, without coming too close to the hot jet shear layer where probe interference effects can contaminate measurements. The near field and far field measurements were not conducted simultaneously recognizing the potential for interference.

Far-field acoustic instrumentation consisted of a fixed 8-microphone array (at normal incidence with grids removed) placed $10 \mathrm{ft}$ from the jet centerline positioned at $10^{\circ}$ increments from $80^{\circ}-150^{\circ}$ relative to the jet inlet axis. The far field microphone array is partially visible in Figure 1. The noise spectra, in the form of narrowband and 1/3-octave band sound pressure 
levels (SPL) from $50 \mathrm{~Hz}-80 \mathrm{kHz}$, are stored together with ambient humidity, pressure, temperature and exhaust conditions. The far-field raw acoustic spectra were first corrected for high frequency microphone response by applying individual microphone frequency calibration corrections. The data was then converted to an ideal, loss-less day (i.e. no attenuation due to humidity). A weather correction is applied to the data to bring it to standard conditions $\left(77^{\circ} \mathrm{F}\right.$ and $70 \%$ relative humidity).

\section{Analysis}

\subsection{Far field acoustics}

The objective of the current study is to sense the pressure signatures of large-scale turbulent structures in the jet near field, and analytically relate their statistical properties to sound radiation. Toward this end, we assume that the near-field microphones are in a region where pressure is governed by the linear wave equation. Thus, we require that the measurement location is sufficiently near the jet such that the pressure field is dominated by hydrodynamic disturbances, yet sufficiently far from the jet such that the effects of non-linearity and mean flow non-uniformity can be reasonably neglected.

We solve the wave equation

$$
\partial^{2} p / \partial t^{2}-c_{0}^{2} \partial^{2} p / \partial x_{i}^{2}=0
$$

with pressure specified on a cylindrical surface of radius $r=r_{0}$ surrounding the jet. This is a simplifying approximation to the slightly conical surface on which pressure data is acquired. Validity of this approximation will be assessed in future studies.

Equation (1) is solved by applying Fourier transforms in the streamwise $(x)$ and azimuthal directions $(\theta)$, and solving the resulting boundary value problem in the radial coordinate with pressure specified at $r=r_{0}$. The inverse transform is evaluated asymptotically in the far field using steepest descents. The jet far field pressure spectral density $S(\omega)$ can then be expressed as

$$
\begin{aligned}
S(\omega)= & \frac{4}{(2 \pi)^{3} R^{2}} \times \\
& \sum_{m} \frac{1}{\left|H_{m}^{(1)}\left(k r_{0} \sin \phi\right)\right|^{2}} \int Q_{m}\left(x, r_{0}, \omega\right) d x
\end{aligned}
$$

where $(R, \phi)$ are the observer polar coordinates, $k=$ $\omega / c_{0}$ is the acoustic wavenumber, and

$$
Q_{m}\left(x, r_{0}, \omega\right)=\left.\hat{P}_{m}\left(x, r_{0}, \lambda, \omega\right)\right|_{\lambda=-k \cos \phi}
$$

where

$$
\begin{aligned}
& \hat{P}_{m}\left(x, r_{0}, \lambda,, \omega\right) \equiv \int P\left(x, r_{0}, \Delta x, \Delta \theta, \Delta t\right) \times \\
& \mathrm{e}^{\mathrm{i} \lambda \Delta x} \mathrm{e}^{\mathrm{i} m \Delta \theta} \mathrm{e}^{\mathrm{i} \omega \Delta t} d(\Delta x) d(\Delta \theta) d(\Delta t)
\end{aligned}
$$
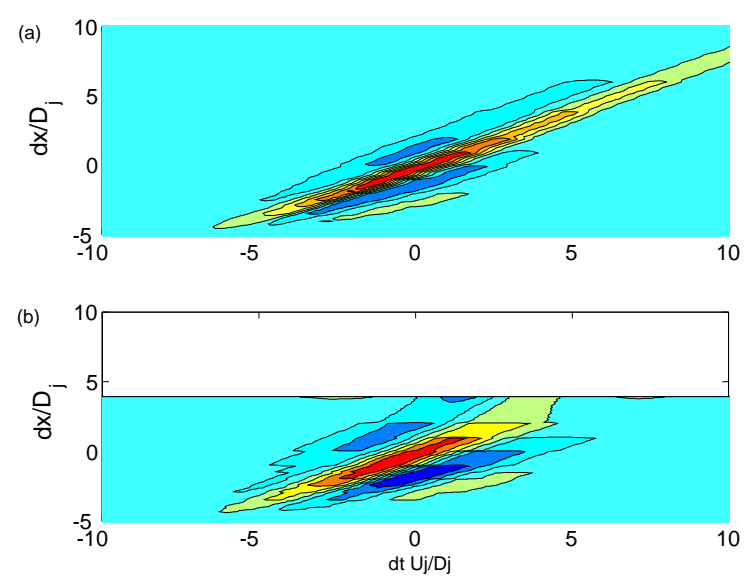

Fig. 4 Pressure correlation measured as function of normalized time-space separation. Reference probe at 6 jet diameters. (a) $M_{j}=0.9$ hot, (b) $M_{j}=0.6$ hot.

is the wavenumber-frequency spectrum of pressure at $r=r_{0} . P$ is the pressure correlation

$$
\begin{aligned}
& P\left(x, r_{0}, \Delta x, \Delta \theta, \Delta t\right)= \\
& \quad\left\langle p\left(x, r_{0}, \theta, t\right) p\left(x+\Delta x, r_{0}, \theta+\Delta \theta, t+\Delta t\right)\right\rangle
\end{aligned}
$$

where we have assumed statistical homogeneity in $\theta$. The function $Q_{m}(x)$ defines an axial source density distribution representing the contribution to $S(\omega)$ from all sources correlated with the reference location $x$. In general, contributions associated with different values of $x$ are correlated, and may combine constructively or destructively to $S(\omega)$. Note also that the relation (3) between the source distribution and the pressure statistics at $r=r_{0}$ corresponds to the well-known result that only spectral components of the source with the acoustic phase relation $\lambda=-\left(\omega / c_{0}\right) \cos \phi$ contribute to far field sound at observer angle $\phi$.

\subsection{Analytical source model}

Typical results for the near-field pressure correlation $P\left(x, r_{0}, \Delta x, \Delta t\right)$ are shown in Figure 4 . The correlation takes the form of a wave packet, having finite extent in space and time, and a pronounced convective phase. To the extent that it represents hydrodynamic events (as opposed to acoustics), the correlation can be interpreted physically as an ensemble-averaged representation of the growth-and-decay cycle of turbulent structures passing the reference probe at $x$. As a simple analytical representation of this process, we consider a model of the form

$$
P(\Delta x, \Delta t)=A(\Delta t) G\left(\Delta x-U_{c} \Delta t\right)
$$

where $U_{c}$ is a constant convection speed and $G(x)$ defines the spatial turbulence structure which, except for modulation by the amplitude function $A(\Delta t)$, is unchanging in the moving reference frame $\Delta x=U_{c} \Delta t$. 


\section{AIAA Paper 2003-3314}

It proves convenient to select the functional forms

$$
A(t)=\exp \left[-\alpha^{2}\left(t-t_{0}\right)^{2}\right]
$$

and

$$
G(x)=G_{0} \exp \left[\mathrm{i} \kappa x-\beta^{2}\left(x-x_{0}\right)^{2}\right]
$$

where the real part of (8) is used in representing the data. Note that the time scale

$$
T \equiv \alpha^{-1}
$$

characterizes the lifetime of the structure, and the length scale

$$
L \equiv \beta^{-1}
$$

characterizes the streamwise correlation scale in the moving reference frame.

Evaluating the wavenumber-frequency spectrum (4) of (6)-(8) and setting $\lambda=-k \cos \phi$, we can write

$$
\left.P(\lambda, \omega)\right|_{\lambda=-k \cos \phi}=\frac{\pi}{2 \alpha \beta} P_{1}(S t) P_{2}(S t)
$$

where

$$
P_{1}(S t)=\exp \left\{-\left(T U_{j} / D_{j}\right)^{2} \pi^{2} S t^{2}\left(1-M_{c 0} \cos \phi\right)^{2}\right\}
$$

and

$$
\begin{aligned}
P_{2}(S t)= & G_{0} \exp \left\{-\frac{\left(\kappa D_{j}+2 \pi S t M_{j 0} \cos \phi\right)^{2}}{4\left(D_{j} / L\right)^{2}}\right\} \\
& +G_{0}^{*} \exp \left\{-\frac{\left(\kappa D_{j}-2 \pi S t M_{j 0} \cos \phi\right)^{2}}{4\left(D_{j} / L\right)^{2}}\right\}
\end{aligned}
$$

where $S t=f D_{j} / U_{j}$. Mach numbers are defined relative to the ambient sound speed.

Two distinguished limits can be noted in the above result. First,

$$
L / D_{j} \ll 1, \quad T U_{j} / D_{j}=O(1)
$$

corresponds to a limit in which the local correlation scale is small compared to the distance $T U_{c}$ traversed by the structure during its lifetime. This is analogous to a convected-singularity jet noise model. In this case, the dependence of $P_{2}(S t)$ on frequency is weak, and behavior with $S t$ in (11) is governed by $P_{1}(S t)$. It can be seen that $P_{1}$ exhibits the classical scaling with a Doppler-corrected frequency.

The second limit we consider is

$$
L / D_{j}=O(1), \quad T U_{j} / D_{j} \ll 1
$$

in which the structure turnover time is rapid, in the sense that the structure does not travel many correlation lengths during its lifetime. In this case, behavior with frequency is governed by $P_{2}(S t)$, where frequency scales with Helmholtz number $S t M_{j 0}$. Experimental results presented by $\mathrm{Lush}^{25}$ have indeed shown that, at aft angles, far field spectra do not exhibit Strouhal number scaling with increasing jet speed, but tend to have a fixed peak frequency, consistent with a $S t M_{j 0}$ scaling. The above result suggests that such behavior may be expected if the structure lifetime $T U_{j} / D_{j}$ is sufficiently small. In Section 4.3, model parameters defined in (6)-(8) are identified by a least-squares fit to experimental data for $P(\Delta x, \Delta t)$, and the effect of time scale $T$ on spectral scaling with jet Mach number is investigated further.

\subsection{DNS data base}

In order to investigate several features of the experimental procedure for estimating $S(\omega)$, we use a well-validated DNS database ${ }^{2}$ for a $M_{j}=0.9$ turbulent cold (uniform stagnation temperature) jet with $R e=3600$. We cite here a few details of the DNS methodology that are relevant to the present study; further details of the numerical method and its validation are described by Freund. ${ }^{2}$

The Reynolds number is very much smaller than that of the experiments and there are certain differences in both the turbulence and acoustic radiation that have an impact on the comparisons made here. First, and foremost, the initial shear layers of the jet are laminar and therefore nearly silent compared to the acoustic sources near the close of the potential core. Thus an important source of realistic jet noise (especially at higher frequencies) is not simulated. The turbulence near and downstream of the potential core, on the other hand, is realistic and the Reynolds stresses match those measured in much higher Reynolds number jets. ${ }^{26,27}$ Moreover, kinetic energy spectra are broadband, two-point velocity correlations decay rapidly in space, and physically resolved dissipation of turbulent kinetic energy is a significant factor in the overall energy budget.

The computational domain was cylindrical and included the near field, up to $16.5 \mathrm{D}$ in the streamwise direction and $4 D$ in the normal direction. A total of $(640,250,160)$ grid points were placed in the $(x$, $r, \theta)$ directions, respectively. The flow was artificially seeded with very small amplitude, solenoidal disturbances in a buffer region near the inflow boundary. The velocity profile and the momentum thickness of the shear layers matched closely the corresponding experiments (at the same Reynolds number) of Stromberg et al. ${ }^{28}$, by $x / D=1$. The far-field sound to arbitrarily large distances from the jet was subsequently computed by using a cylindrical Kirchhoff surface at $r=4 D$, ignoring any contribution from the open ends. The resulting acoustic field is contaminated at very shallow angles to the jet axis (less than about 20 degrees). The sound field was computed in two different ways from the data on the Kirchhoff surface: a Fourier method and a direct finite-difference solution of the wave equation, giving nearly identical results. 


\section{AIAA Paper 2003-3314}

In the course of the simulation, all flow variables were saved every 20 numerical time steps of $(\Delta t)_{D N S}=0.0085 r_{o} / a_{\infty}$ on every other mesh point in space. This resolution is sufficient to compute most flow quantities. There are, in all, 2333 different instants in time saved after the jet has reached a statistically steady state. Such a sample size is certainly sufficient for low-order statistics. For the frequency spectra, the sampling rate is sufficient to obtain a good representation of the highest frequencies at this particular Reynolds number. However, it should be noted that the total sample length gives a rather course binning of the frequency spectra, and is not sufficient to completely converge the statistics of the lowest frequencies. Moreover, due to the relatively low Reynolds number, the acoustic field is more tonal. Thus while the pressure correlation given by equation (5) is fairly well converged, the presence of slowly decaying tails with $\Delta t$ necessitates the need for windowing the correlation prior to taking the Fourier transform (4). We use a maximum time delay of $\Delta t=68 r_{0} / a_{\infty}$ and choose a window similar to Freund, ${ }^{2}$ where the window is unity over the middle $90 \%$ of the data, and falls to zero (using tanh functions) over the first and last $5 \%$ of the data. In what follows, spectra have also been binned by smoothing (running averages of nearby values) prior to plotting (but not in the processing).

For simplicity, we restrict our investigation to the pressure fluctuations in the zeroth azimuthal mode $(n=0)$. The acoustic field is dominated by sound from the first few azimuthal modes, and spectra and directivity are not significantly different for the different modes. In the experimental projection from near-jet pressure correlations to far-field sound, the limited microphone array necessitates that some assumption is made regarding the azimuthal spectrum of disturbances-in the present analysis, we have assumed that the experimental pressure fluctuations are dominated by mode $n=0$.

\section{Results and Discussion}

\subsection{Analysis of DNS data}

As discussed in section 3.1, the correlation projection method requires that the pressure fluctuations at microphone positions are approximately governed by the linear wave equation. The pressure fluctuations may be dominated by evanescent (hydrodynamic) waves, but we require that mean flow gradients and nonlinear effects are small. In Freund, ${ }^{2}$ Lighthill's tensor, $T_{i j}$ and its statistics were computed. Wherever $T_{i j}$ is linear, density fluctuations are governed by the wave equation. Provided that entropy fluctuations are not significant (the flow is cold), then pressure fluctuations are governed by the wave equation wherever density fluctuations are. Based on the contour maps in Freund, ${ }^{2}$ we estimate the maximal streamwise distance, at any given distance from the centerline, where the pressure fluctuations are governed by the wave equation. For selected radii, the results are tabulated in Table 1.

\begin{tabular}{c|c} 
Cylinder radius, $r_{0}$ & Distance downstream, $x$ \\
\hline $0.5 D$ & 0 \\
$0.75 D$ & $2.5 D$ \\
$1 D$ & $6 D$ \\
$1.5 D$ & $8 D$ \\
$2 D$ & to end \\
$3 D$ & to end \\
$4 D$ & to end
\end{tabular}

Table 1 The maximal streamwise location where, for a given distance from the jet centerline, pressure fluctuations are governed by the wave equation.

In Figure 5, we plot spectra in the far field at an aft angle of 150 degrees (near which overall SPL is maximum). Results are plotted using both the original Kirchhoff surface (wherein the wave equation was solved for $r>4 D_{j}$ ), and the present projection of pressure correlations (i.e. $S(\omega)$ computed with equation 2) with different values of $r_{0}$. Good agreement is obtained between the two methods provided $r_{0}$ is greater than about $2 D_{j}$, which is consistent with the estimates of Table 1 . The spectrum is slightly overestimated when $r_{0}=2 D_{j}$ and $3 D_{j}$. This is likely due to the signal processing issues mentioned previously-at smaller radii the pressure correlations are dominated by hydrodynamic (evanescent) waves. In computing the Fourier transform in $x$ (see equation 4 ) for the wavenumber at $\lambda=-\omega / c_{0} \cos \phi$, there may be leakage from the (far greater) peak at $\lambda=-\omega / U_{c}$, where $U_{c}$ is the dominant convection speed of the large scale structures. This effect is strongest for the low frequencies, i.e. $S t_{D} \approx 0.1$, where one expects the broadest spatial correlation. However, even when the surface intersects the jet, as for example with $r_{0}=1.5 D_{j}$ and $r_{0}=D_{j}$, we find that the estimate for $S(\omega)$, near the peak at $S t_{D}=0.2$, is only overestimated by a factor of about 2. The spectra at $r_{0}=1.5$ has qualitatively the same shape as the true far-field spectra, while at $r_{0}=1 D_{j}$, the higher frequencies are significantly over-estimated. Again, based on the analysis, we only expect strict agreement for $r_{0}>2 D_{j}$.

Aside from agreement with previous far-field computations, another assessment of the quality of the data and post-processing can be obtained by comparing the real and imaginary parts of the obtained spectrum, $S(\omega)$. In the absence of errors, the imaginary part of $Q_{x}$ must integrate to exactly zero, since by definition the left-hand-side of equation 2 is real. Moreover, the real part must integrate to a positive number since $S(\omega)$ is positive. The symmetries that guarantee this are not respected by the discretized signal processing and therefore the extent to which there is an imag- 


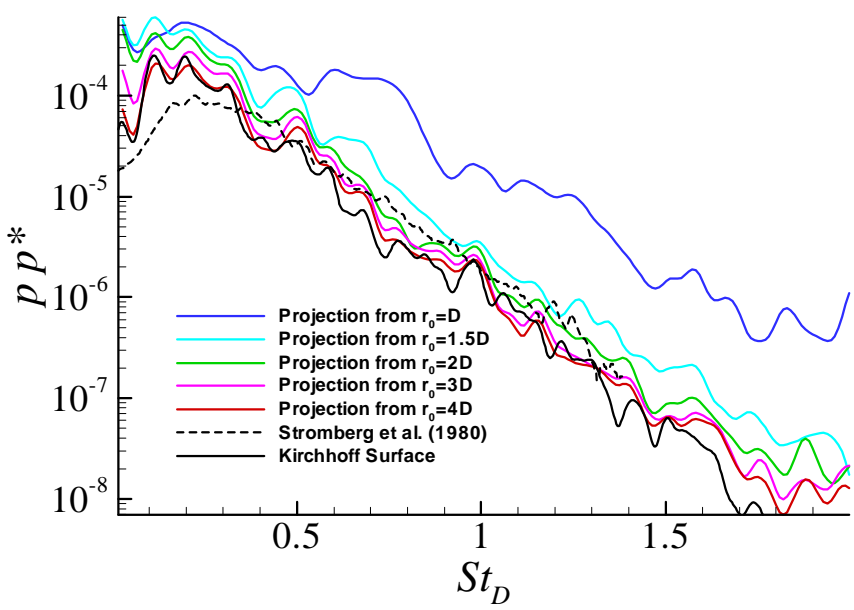

Fig. 5 Projected far-field pressure spectra $(S(\omega))$ at $30 D_{j}$ and 150 degrees as a function frequency, $S t_{D}$ using different values of $r_{0}$. Also plotted is the spectra computed directly from the previous Kirchhoff surface method from $r_{0}=4 D_{j}$ and the experimental data of. ${ }^{28}$

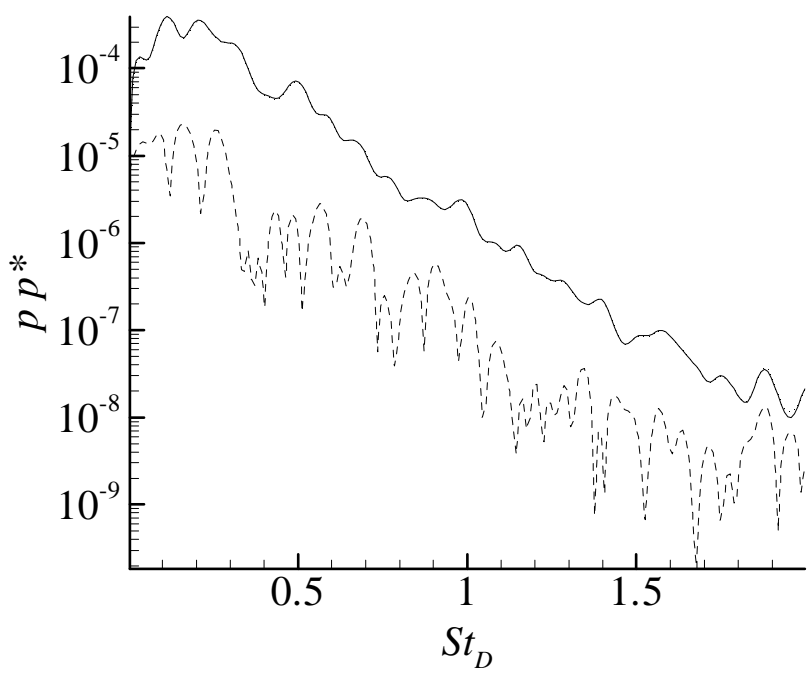

Fig. 6 Projected far-field pressure spectra $(S(\omega))$ at $30 D_{j}$ and 150 degrees as a function of frequency, $S t_{D}$ using $r_{0}=4 D_{j}$. The dashed line is the absolute value of the imaginary part, and the dotted and solid lines (which are coincident) are the real part and magnitude, respectively.

inary component (or a negative real result) gives an assessment of the overall error of the procedure. The real part, imaginary part, and magnitude of $S(\omega)$ for $r_{0}=4 D_{j}$ are plotted in Figure 6 . The imaginary part is about a factor of 10 smaller than the real part, and the real part is always positive.

Pressure correlations at several values of $r_{0}$ at $x=$ $10 D_{j}\left(3 D_{j}\right.$ downstream of the potential core end $)$ are
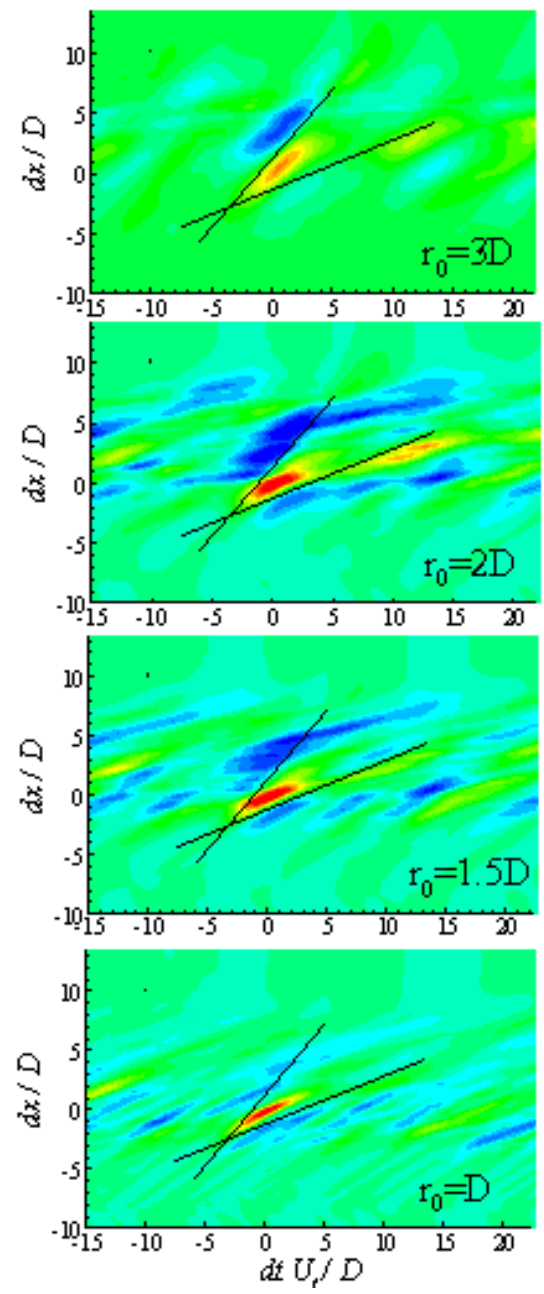

Fig. $7\left\langle p\left(x, r_{0}, n=0, t\right) p\left(x+\Delta x, r_{0}, n=0, t+\Delta t\right)\right\rangle$ at $x=10 D$ and different values of $r_{0}$. The superimposed lines mark a convection speed of $0.4 U_{j}$ (shallower line), and the $x$-component of acoustic propagation to 150 degrees (maximum aft radiation angle).

shown in Figure 7. These show, for $r_{0}=D_{j}, 1.5 D_{j}$, $2 D_{j}$ that the pressure fluctuations are indeed dominated by hydrodynamic evanescent waves. The average phase speed of the correlation is close to $0.4 U_{j}$, which is consistent with the location downstream of the potential core (and with values computed by Freund $\left.^{2}\right)$. By $r_{0}=3 D_{j}$, on the other hand, the correlation is acoustic, with a phase speed very close to that for the dominant acoustic radiation to 150 degrees.

So far we have shown that reasonable results can be obtained for the total spectrum even when $r_{0}$ is taken as small as $1.5 D_{j}$. The principle question that must be addressed in order to assess the validity of the projection method for experimental data is: to what extent can we minimize the number of reference microphone locations (i.e. locations where $Q_{m}(x)$ is measured), and still obtain significant information about the acoustic field, at least at the peak frequen- 
cies which are dominated by large-scale structures. The answer will depend largely on the spatial extent of the source region, and the extent to which there is mutual cancellation between positive and negative contribution (i.e. of opposite phase).

In order to address this, $Q_{0}\left(x, r_{0}, \omega\right)$ is plotted for several values of $r_{0}$ in Figure 8 . We concentrate on the frequencies around $S t_{D}=0.1$ to 0.5 . Note that the contour levels are identical for all plots. The levels of $Q_{0}$ have been normalized by dividing by the peak value of $S(\omega)$ at $30 D$ and 150 degrees.

For $r_{0}=3 D_{j}$ (where the signal is already largely acoustic) we find, as one might expect, that there are only positive contributions and the integrand is very flat, with a spatial extent of about $4-5 D_{j}$. The flat nature is indicative that any cancellations have already occurred. For $r_{0}=2 D_{j}$, where the signal is largely hydrodynamic, the integrand is also quite flat, and has only small canceling negative regions upstream of the dominant values. The spatial extent is more compact, and centered about $x=11 D_{j}$ for $S t=0.1$ and $x=9 D_{j}$ for $S t=0.2$. By $r_{0}=1.5 D_{j}$, the negative "tails" have increased somewhat, and the positive region has split into two 'bumps', but $Q_{0}$ remains reasonably flat. Finally, at $r_{0}=D_{j}$, there are larger and more intense negative regions, and the positive region is more peaked. However, it should be kept in mind that both of the latter radial positions are within the jet, where the analysis is not valid and the integrated values for $S(\omega)$ are significantly over-predicted.

As might be expected, when higher frequencies and angles less than about 130 degrees are examined, one finds broader and considerably more oscillatory regions where $Q_{0}$ is nonzero for $r_{0}<3 D_{j}$. The higher frequencies are less directional, and probably arise from a much broader region of the jet downstream of the potential core.

We conclude that provided the microphone locations are in a region where the wave equation is valid and reasonably close to the source of peak frequency radiation, then relatively few reference microphone positions would be required to obtain an estimate of the acoustic field, at least at aft angles and near the peak frequencies. The more that is known about the extent of the source region, the fewer positions that would be required. In the extreme case, if it is known that the source extent for $S t_{D}=0.2$ is about 5 diameters, and we place a single microphone at $x=10 D_{j}, r_{0}=2 D_{j}$, then we merely multiply $Q_{0}(10 D, 2 D, \omega)$ by 5 to obtain the far-field spectra. In more realistic cases, of course, it would likely require an array of near field microphones, but because $Q_{0}$ is flat within the source region, they can be relatively sparsely spaced. One could in principle shift the reference probe upstream or downstream slightly (and perhaps in the radial position) to test the flatness of $Q_{0}$ in order to ensure that the array is not too close to the jet.
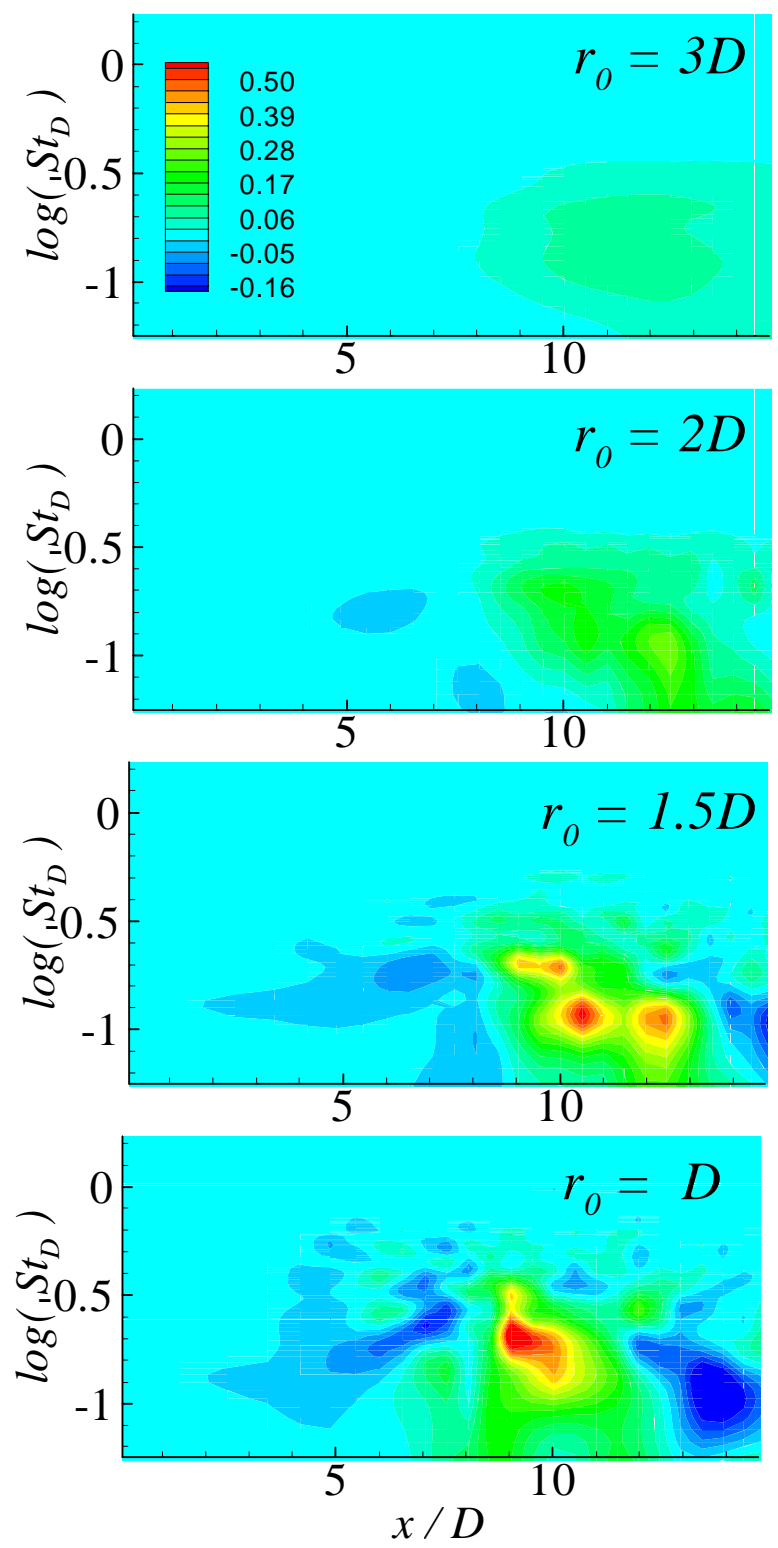

Fig. $8 Q_{0}\left(x, r_{0}, \omega\right)$, as a function of reference position, $x$, and frequency, $S t_{D}$. The integrated value of $Q_{0}$ over all $x$ produces the spectra plotted in figure 5 The contour levels are shown in the plot and are identical for all values of $r_{0}$ shown.

\subsection{Analysis of experimental data}

We first analyze the experimental data for dynamic pressure acquired in the high-speed, hot jet near field to illustrate the underlying organized structure dynamics of interest. Figure 9 displays the power spectra from the near field microphone that was traversed along the jet shear layer (outer) edge for $M_{j}=0.6,0.9$ hot jets. A smaller streamwise extent of $x / D_{j} \leq 10$ is displayed for the Mach 0.6 hot jet, whereas results for a larger spatial extent $x / D_{j} \leq 20$ are shown for the Mach 0.9 hot jet. The organization of the dynamics 

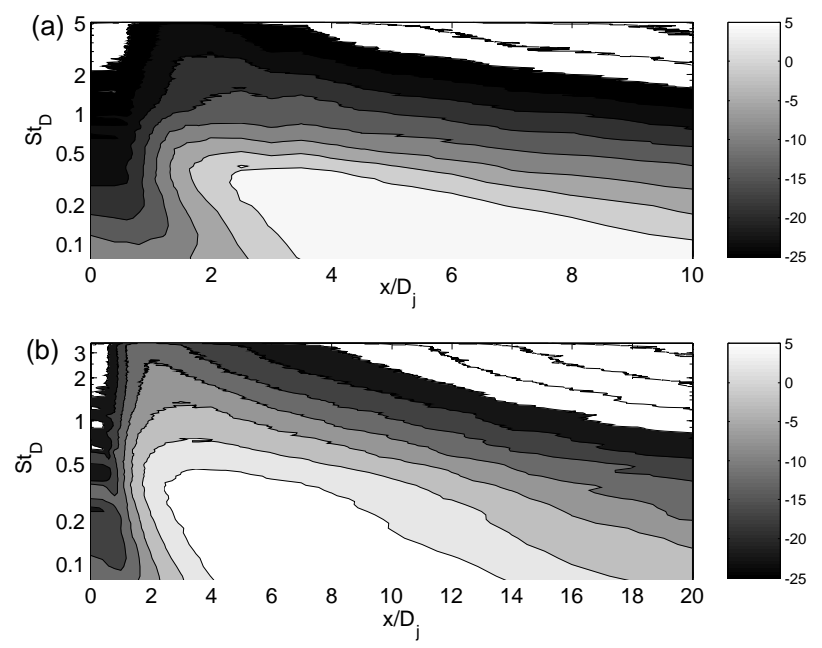

Fig. 9 Power spectra from near field pressure measurements showing contour levels of the sound pressure levels (no calibration applied) as a function of the non-dimensional frequency $\left(S t_{D}\right)$ and the streamwise location of the probe for: $M_{j}=\mathbf{0 . 6}$ (a) and 0.9 (b), with $T_{j}=1000 \mathrm{~F}$ for both cases; note that the Mach numbers quoted are relative to local sound speed (inside heated jet).

in a broadband surrounding high frequencies near the nozzle exit plane and lower frequencies as one moves downstream is evident in Figure 9a,b. This is to be expected based on the growing scales of structures in the jet shear layer, whose imprint is captured by the near field probe. The frequencies have been normalized to $S t_{D}=f D_{j} / U_{j}$ with the respective jet velocities $U_{j}$ and jet diameter $D_{j}$. Recall that one of the two fixed reference probes was positioned close to the jet potential core end for the two-point correlation measurements. Judging from the power spectra it is evident that close to this reference location frequencies around $S t_{D}=0.3$ are most dominant, which corresponds to the well-known jet column mode. ${ }^{7}$ Capture of lower frequency dynamics using two-point correlation measurements would therefore be feasible only when the reference probe is moved farther downstream; this will be further addressed in a later section.

Figure 10 displays the coherence spectra for a range of traversing probe locations relative to a fixed reference probe located at $x / D_{j}=6$. The peak coherence levels are seen for $S t_{D} \sim 0.3$ close to the reference probe location where the two probes are closest to each other (although circumferentially separated by $90^{\circ}$.). To the extent that the measurements capture hydrodynamics (as opposed to acoustics), the distributed axial extent is indicative of the length scale associated with the organized structures in the jet. (Acoustic vs. hydrodynamic content of the data is discussed below). Measurements of relative phase between azimuthally separated probes at or near $S t_{D} \approx 0.3$ in this region indicate three-dimensional coherent dynamics. How-

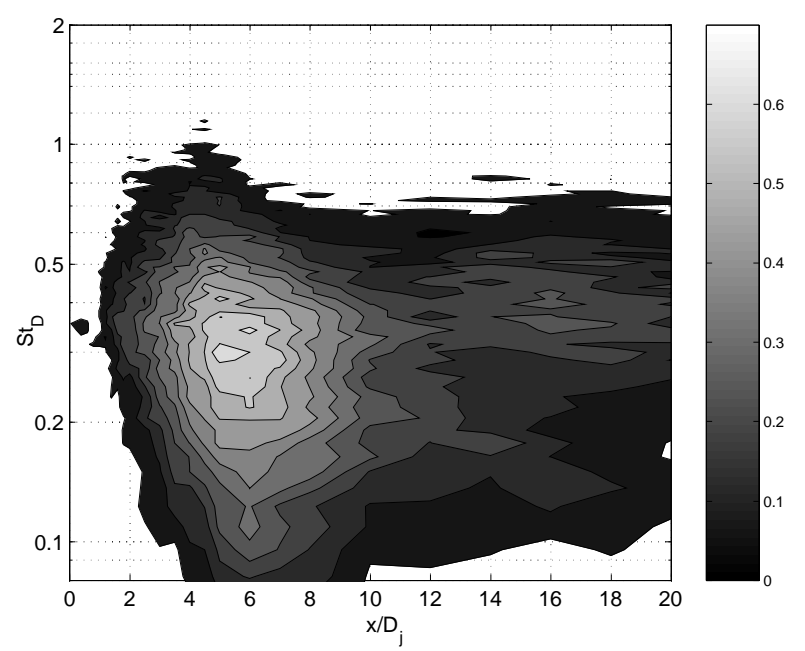

Fig. 10 Coherence spectra from near field pressure measurements for $M_{j}=0.9, T_{j}=1000 \mathrm{~F}$, showing contour levels of the coherence as a function of the non-dimensional frequency $\left(S t_{D}\right)$ and the streamwise location of the probe; fixed reference probe at $x / D_{j}=6$.

ever, we neglect the three-dimensional behavior in the analysis for now, and assume that $m=0$ is dominant.

Space-time correlation data and analysis. In the following discussion we first analyze the experimental data to determine the extent to which the measured near-field pressure represents hydrodynamic sources of sound, as opposed to sound itself. Near-field pressure correlations were shown in Figure 4 for $M_{j}=0.6$ and $M_{j}=0.9$ heated jets at $T_{0}=1000 \operatorname{deg} F$. The jet velocities are $U_{j}=329$ and $474 \mathrm{~m} / \mathrm{s}$, respectively, and the reference probe is located at $6 D_{j}$. Probe separation is normalized by $D_{j}$, and time delay is normalized by $D_{j} / U_{j}$. The correlations collapse reasonably well when scaled with jet velocity, suggesting that the pronounced convective ridge is associated with hydrodynamic disturbances. This is further illustrated in Figure 11, where the location of peak correlation is plotted in the time-delay/axial-separation plane. The convective ridge is seen to collapse reasonably well along a line corresponding to $0.7 U_{j}$, although the convection speed increases with probe separation. This effect is most pronounced in the $M_{j}=0.6$ case, where the disparity between acoustic speed and typical turbulence convection speeds is largest. This behavior suggests that the correlation becomes dominated by acoustic disturbances when the moving probe is more than 1-2 jet diameters downstream of the reference probe. This conclusion is further supported by the peak correlation amplitude shown in Figure 12, where pressure is scaled by the hydrodynamic scale $\rho_{j} U_{j}^{2}$. The amplitude is seen to collapse fairly well for locations upstream of the reference probe, but the collapse is relatively poor downstream, with the correlation at higher Mach number tending to persist in the down- 


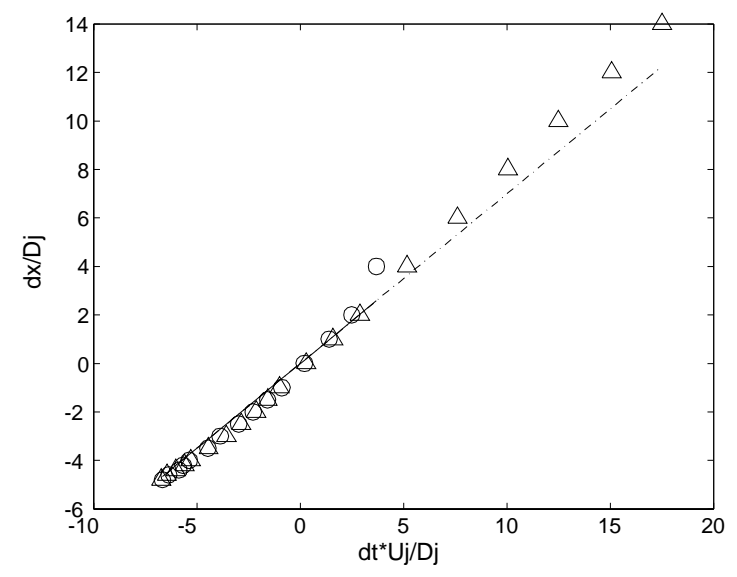

Fig. 11 Location of correlation peak in normalized time-space separation plane. $M=0.9$ hot (triangles) and $M=0.6$ hot (circles). Reference probe at 6 jet diameters.

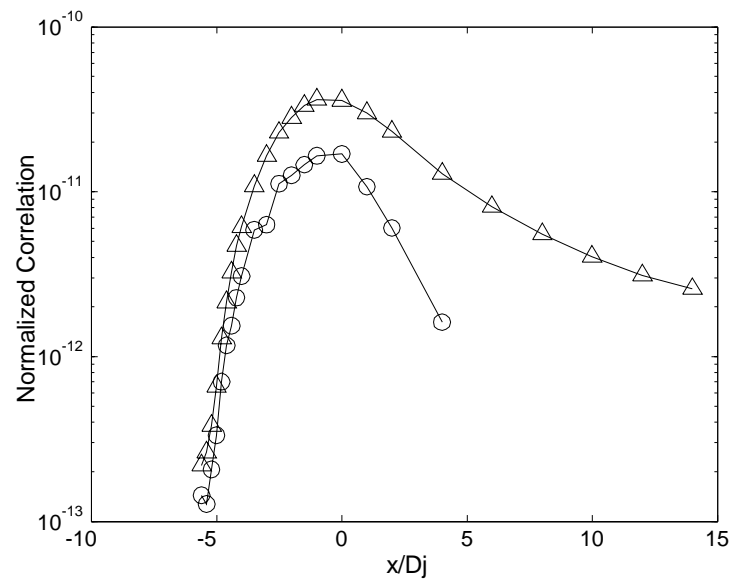

Fig. 12 Peak correlation amplitude normalized by hydrodynamic pressure scale as function of axial separation. $M=0.9$ hot (triangles) and $M=0.6$ hot (circles). Reference probe at 6 diameters.

stream direction, presumably due to the higher relative amplitude of acoustic disturbances.

Pressure cross-spectra with pressure normalized by the hydrodynamic scale $\rho_{j} U_{j}^{2}$ are shown in Figures 13-14 for the two jet operating conditions described above. For the moving-probe location of $x / D_{j}=3.5$, the cross-spectra collapse quite well for $S t<0.4$. At higher frequencies, the pressure spectra exhibit scaling with a much higher power of velocity (in the range of $\left.U_{j}^{7-8}\right)$. These results suggest that pressure fluctuations at the lower frequencies are indeed signatures of hydrodynamic disturbances, while the higher frequencies are acoustic in nature. For the moving probe location of $x / D_{j}=7.0$, the cross-spectra collapse with the hydrodynamic pressure scale over a narrower frequency range. This result is consistent with the prior conclusion that the correlation for probe locations downstream of the reference probe tend to be

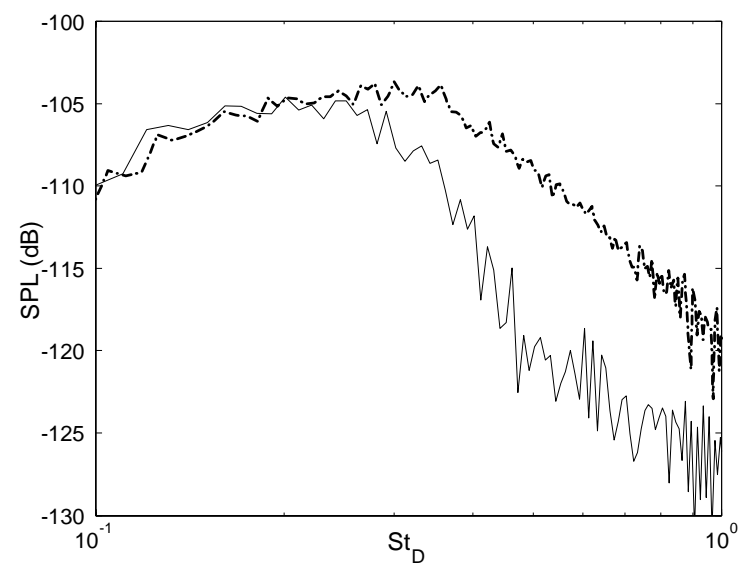

Fig. 13 Cross-spectra normalized by hydrodynamic pressure scale. $M=0.9$ hot (dotted) and $\mathrm{M}=0.6$ hot (solid). Reference probe at 6 jet diameters and moving probe at 3.5 diameters.

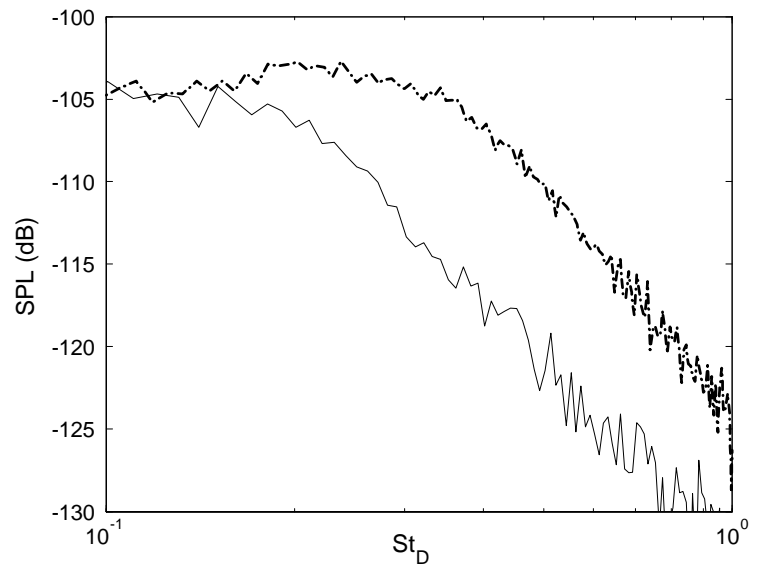

Fig. 14 Cross-spectra normalized by hydrodynamic pressure scale. $M=0.9$ hot (dotted) and $\mathrm{M}=0.6$ hot (solid). Reference probe at 6 jet diameters and moving probe at 7 diameters.

dominated by acoustic disturbances.

Far field sound computed by applying (2) to the pressure correlation for $x_{r e f}=6 D_{j}$ at $M=0.9$ is shown in Figure 15. It has been assumed here that the pressure field consists entirely of the axisymmetric mode $m=0$. Also, the source density (3) has been multiplied by a weighting factor of $12 D_{j}$, selected to best agree with the far field data. Results are shown for observer angles of 130 and 150 degrees. At 150 degrees, the far field sound computed from the near-field correlation agrees reasonably well in terms of spectral shape with data at Strouhal numbers greater than about 0.3 . The discrepancy at lower frequencies is believed to arise from the neglect of large-scale structures downstream of $6 D_{j}$, which are not captured by the correlation with reference probe at $6 D_{j}$. It is also seen that the overall trend in directivity is fairly well cap- 


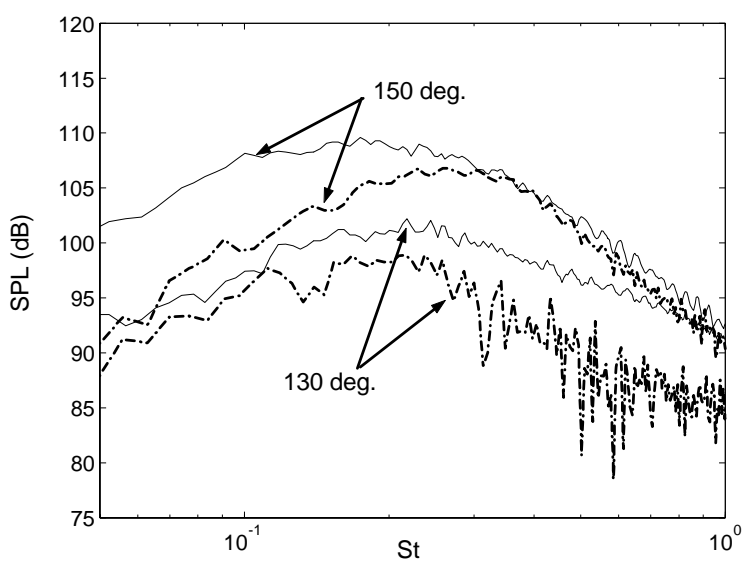

Fig. 15 Comparison of measured far field pressure spectra (solid) with far field projection (dashed) of near field data. $M=0.9$ hot jet.

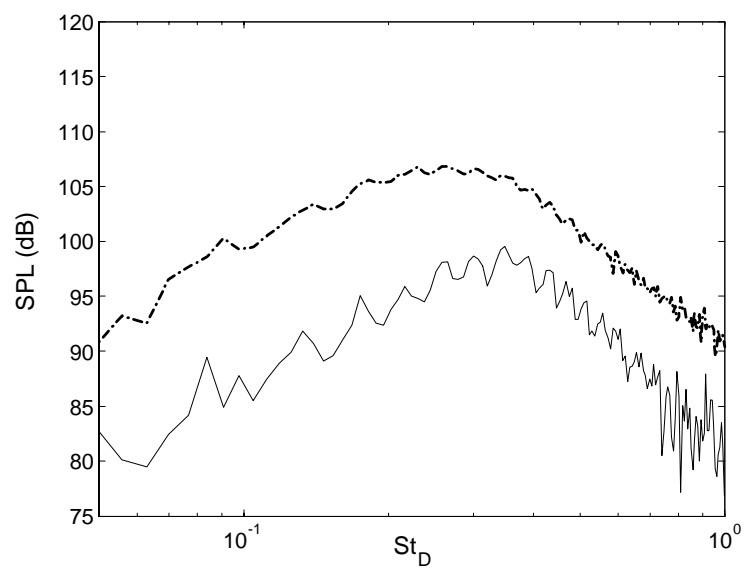

Fig. 16 Contribution to far field pressure from source density at 6 jet diameters (dotted) and 2 diameters (solid). $M=0.9$ hot jet.

tured. At the more sideline angle of 130 deg., however, the spectrum decays rapidly at higher frequencies, consistent with the fact that contributions from finer-scale turbulence are not captured by these measurements.

The need for a relatively large source density weighting factor of $12 D_{j}$ suggests that the reference microphone may be situated somewhat away from the region of peak source density. Still, the level of agreement, in an order-of-magnitude sense, is encouraging, and the factor $12 D_{j}$ is not dramatically larger than the source extent of $5 D_{j}$ found earlier in the DNS.

The far field projection has also been done for the $M_{j}=0.6$ hot jet. In this case, a weighting factor larger by roughly an order of magnitude was required. The source of this discrepancy is being investigated further.

Contributions to the far field sound from the reference microphone at $2 D_{j}$ and $6 D_{j}$ are compared in Figure 16. As expected, the spectrum associated with the reference probe at $2 D_{j}$ peaks at a higher frequency. However, the contribution from $2 D_{j}$ is $5-10 d B$ be-
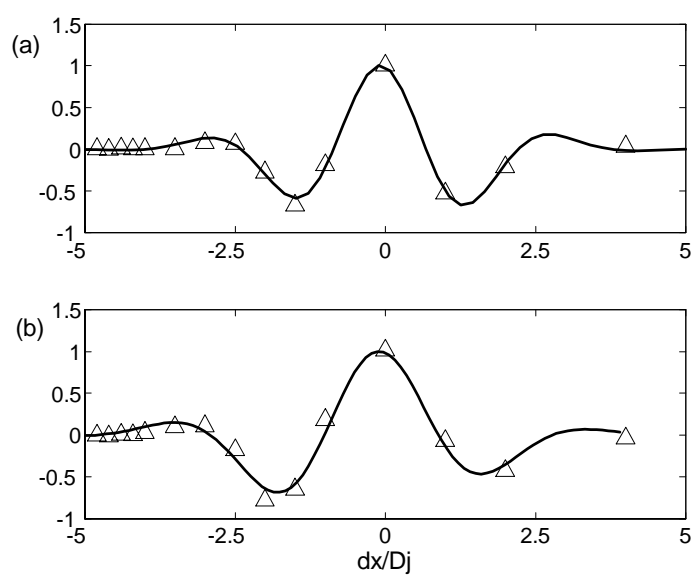

Fig. 17 Model function $G$ compared with data. (a) $M_{j}=0.9$ hot, (b) $M_{j}=0.6$ hot.

low that from $6 D_{j}$, suggesting that the contribution from structures in the initial mixing region is relatively weak.

\subsection{Source model}

In this subsection we determine source model parameters in (6)-(8) by a least squares fit to the experimental data, and consider the effect of turnover time scale $T$ on frequency scaling of far field spectra with jet speed. Note that the model assumes that the spatial shape function $G$ is independent of time, and that the convection speed $U_{c}$ is constant. These are only approximations, and it can be seen in Figure 11, for example, that the convection speed is in fact increasing with downstream distance. Figure 4 shows that the spatial length scale is also increasing slightly with downstream distance. The effect of these features will be considered in future work.

The shape function $G(x)$ is obtained by taking a cut through $\Delta t=0$, and the amplitude $A(t)$ is taken along a line $\Delta x=.7 U_{j} \Delta t$, approximately following the correlation peak. Comparisons of the model functions with data are shown in Figures 17 and 18. It can be seen that the data is well represented by the simple Gaussian functions (7) and (8). Comparisons of far field pressure computed from the measured and modeled correlation are shown in Figure 19. The model is seen to capture spectral shape and amplitude reasonably well at lower frequencies, but departs dramatically from data at higher frequencies. This may be due in part to the assumption of constant convection speed in the model, while the data exhibits some increase of convection speed with downstream distance. This trend will increase energy in the radiating range of the wavenumber-frequency spectrum, and will result in largest discrepancies at higher frequencies, where the acoustic wavenumber moves further from the dominant wavenumber associated with the convective ridge.

We next apply the model for $P(\Delta x, \Delta t)$ derived 
AIAA Paper 2003-3314

(a)

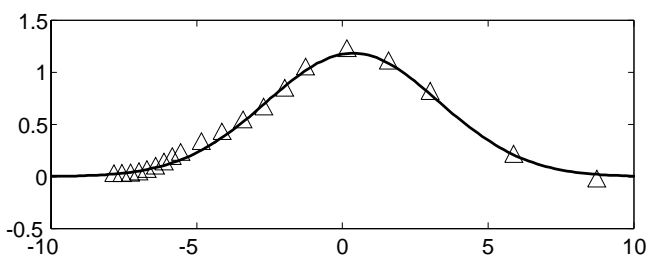

(b)

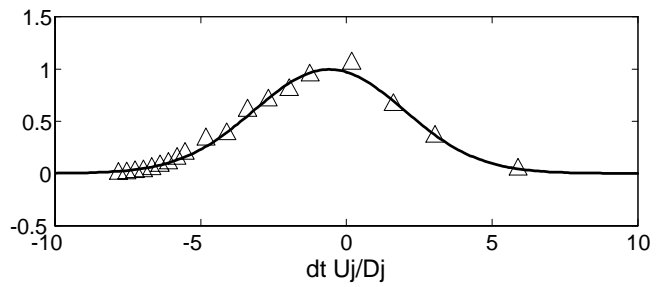

Fig. 18 Model function A compared with data. (a) $M_{j}=0.9$ hot, (b) $M_{j}=0.6$ hot.
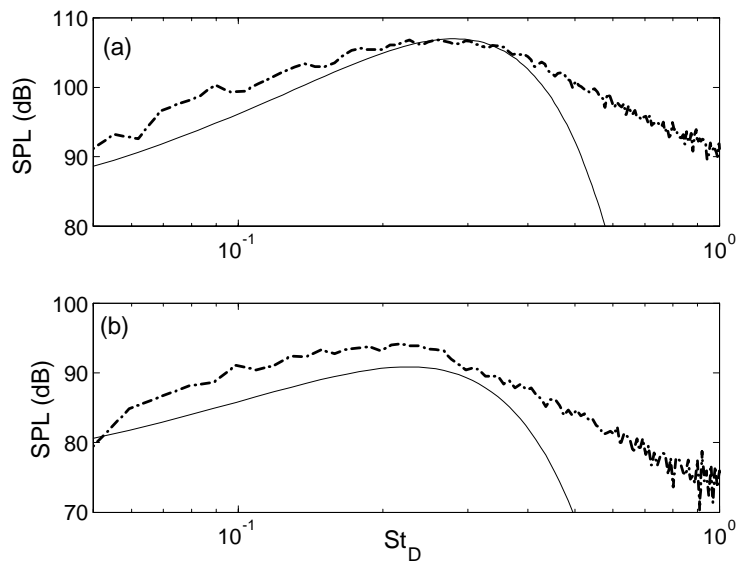

Fig. 19 Comparison of far field spectra at $150^{\circ}$ projected from correlation model (solid) and from data (dashed) for a.) $M_{j}=0.9$, b) $M_{j}=0.6$.

from the $M_{j}=0.9$ hot data to investigate the influence of the time scale (9). We assume that the correlation $P(\Delta x, \Delta t)$ exhibits purely hydrodynamic scaling, so that

$$
P(\Delta x, \Delta t)=\left(\rho_{j} U_{j}^{2}\right) \tilde{P}\left(\Delta x / D_{j}, \Delta t U_{j} / D_{j}\right)
$$

where $\tilde{P}(\xi, \tau)$ is assumed to be a "universal" function independent of Mach number. The dependence on Mach number therefore enters only through evaluation along the sonic line $\lambda=-k \cos \theta$ in the wavenumberfrequency spectrum of $P$. Figure 20 shows far field spectra for $T U_{j} / D_{j}=1$ for three values of jet Mach number. It can be seen that the peak Strouhal number decreases with increasing jet speed, consistent with the results observed by Lush. ${ }^{25}$ This value of $T U_{j} / D_{j}$ corresponds to approximately $1 / 4$ of that computed from the $M=0.9$ hot data. Keeping in mind that the turnover time extracted from the $M_{j}=0.9$ hot data is exaggerated by the significant acoustic content in the correlation with downstream microphones,

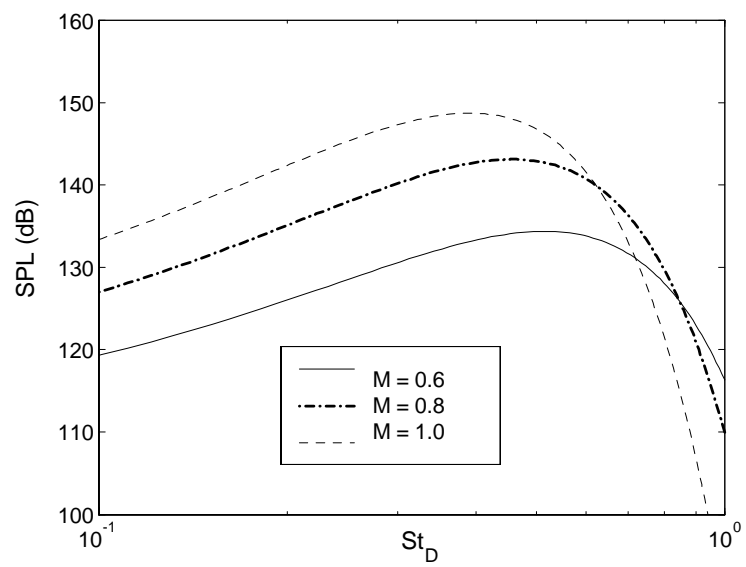

Fig. 20 Farfield spectra computed from analytical source model for $M_{j}=0.6,0.8,1.0$.

and that the turnover time $T$ associated with purely hydrodynamic events may be appreciably smaller, we see that the tendency for far field spectra to scale with Helmholtz number as predicted by the model (6)-(8) may occur for parameters typical of real jets.

\section{Summary and Conclusions}

An analysis framework has been presented for aftangle noise generation by large-scale organized structures in jets. The analysis approach attempts to relate measured near-field pressure correlations to far field sound by applying the linear uniform-medium wave equation. We require, therefore, that the measurement location is sufficiently near the jet for signatures of hydrodynamic disturbances to be dominant, yet sufficiently far from the jet such that non-linear effects can be neglected. The degree to which these conditions can be satisfied was investigated by interrogating DNS data for a Mach 0.9 jet flow. The DNS showed that for near peak frequencies, the far-field spectrum could be estimated, within a multiplicative constant, by finding the pressure correlation at a reference microphone location within about $\pm 2.5 D_{j}$ of the apparent source (which was about $3 D_{j}$ downstream of the mean close of the potential core). It appears that a fairly sparse array of reference positions could also roughly determine the multiplicative constant.

Preliminary results have demonstrated the feasibility of capturing pressure signatures of low-frequency hydrodynamic disturbances in the jet near field at high-speed, heated flow conditions of practical relevance. Results show that, for low frequencies (namely, $\left.S t_{D}<0.4\right)$, the near-field correlations are dominated by hydrodynamic disturbances, while higher frequencies appear to be acoustic in nature. Also, the correlations tend to be dominated by acoustics with increasing downstream distance from the reference microphone. The near-field pressure correlation has been related to far field sound assuming validity of the linear 
wave equation outside the radius of measurement. The far field pressure computed from near field data shows an encouraging level of agreement with the measured far field pressure. Comparison of far field projections for reference microphone locations at 2 and 6 jet diameters suggest that the aft angle spectral contribution from organized structures in the initial jet mixing region are relatively unimportant, even at higher frequencies.

A simple analytical model has been developed for the measured pressure correlations. An ansatz consisting of Gaussian wave packets is shown to provide a reasonable approximation to the data. The resulting model characterizes the source in terms of a convection speed, local spatial correlation scale, and structure lifetime. The parametric model has been used to investigate scaling of far field spectra with jet speed. Results show that, when the local correlation scale is small compared to the distance traversed by the structure during its lifetime, classical scaling with Dopplercorrected Strouhal number is recovered. When the structure time is short, in the sense that it travels relatively few correlation lengths during its lifetime, the far field spectrum tends to scale with Helmholtz number $S t M_{j}$.

The semi-empirical modeling approach presented here offers a promising framework for analyzing sound generation from organized structures in a parametric fashion. This will aid in a better understanding of the sensitivity of the sound field to changes in the flow structure evolution. Future assessments of such semiempirical models will include increasing the number of reference probe locations included in the projection to the far field, and more accurately accounting for the (three-dimensional) jet azimuthal structure. Such assessments can also be used to, for instance, identify the distinguishing effects of jet noise reduction devices (e.g. tabs and chevrons) on the near field structure evolution and noise generation.

\section{Acknowledgements}

This work is jointly funded by United Technologies Research Center and Pratt \& Whitney. The authors would like to thank R. Schlinker and W. Lord for their support and fruitful discussions, Dr. Takao Suzuki for his assistance with signal processing of the DNS data, and Prabir Barooah and Keith Post for their assistance in acquiring the experimenatal data. The authors are grateful to Prof. Jon Freund for access to his database for the turbulent Mach 0.9 jet.

\section{References}

${ }^{1}$ Lighthill, M.J., "On sound generated aerodynamically I. General theory", Proc. Royal Soc., Vol. A221, pp.564-587, 1954.

${ }^{2}$ Fruend, J., "Noise sources in a low-Reynolds-number turbulent jet at Mach 0.9", J. Fluid Mech., Vol. 438, pp.277-305, 2001.
${ }^{3}$ Tam, C.K.W., "Jet Noise: Since 1952", Theor. Comp. Fluid Dynamics, Vol. 10, p. 393, 1998.

${ }^{4}$ Constantinescu, G.K. and Lele, S. K. "Large eddy simulation of a near sonic turbulent jet and its radiated noise," 39th Aerospace Sciences Meeting \& Exhibit, January 8-11, Reno, NV, AIAA Paper-2000-0376, 2000.

${ }^{5}$ Narayanan, S., Noack, B.R. and Meiburg, E., "Reduced order dynamical modeling of sound generation from a jet," 40th Aerospace Sciences Meeting \& Exhibit, January 14-17, Reno, NV, AIAA Paper 2002-0073, 2002.

${ }^{6}$ Soteriou, M., Reba, R. and Maeder, T., "Numerical study of the impact of streamwise vorticity on noise generation by jet flows," 8th AIAA/CEAS Aeroacoustics Conference, June 17-19, Breckenridge, CO, AIAA Paper 2002-2480, 2002.

${ }^{7}$ Hussain, F., "Coherent structures and turbulence", J. Fluid Mech., Vol. , pp. , 1986.

${ }^{8}$ Huerre, P. \& Crighton, D.G., "Sound generation by instability waves in a low Mach number jet", AIAA 8th Aeroacoustics Conference, April 11-13, Atlanta, GA, AIAA-83-0661, 1983.

${ }^{9}$ Crighton, D.G. \& Huerre, P., "Shear-layer pressure fluctuations and superdirective acoustic sources", J. Fluid Mech., Vol. 220, pp. 355-368, 1990.

${ }^{10}$ Laufer, J. \& Yen, T., "Noise generation by a low-Machnumber jet", J. Fluid Mech., Vol. 134, pp. 1-31, 1983.

${ }^{11}$ Morris, P.J. \& Tam, C.K.W, "On the radiation of sound by instability waves of a compressible axisymmetric jet", in Mechanisms of Sound Generation in Flows (ed. E.A. Muller), Springer, 1979.

${ }^{12}$ Mollo-Christensen, E., "Jet noise and shear flow instability seen from an experimenter's viewpoint", American Society of Mechanical Engineers Journal of Applied Mechanics, Vol. 89, pp. 1-7, 1967.

${ }^{13}$ Zaman, K.B.M.Q., "Flow field and near and far sound field of a subsonic jet", J. Sound and Vibration, Vol. 106(1), pp. 1-16, 1986.

${ }^{14}$ Siddon, T.E., "Noise source diagnostics using causality correlations", AGARD CP 131, Noise Mechanisms, pp. 7-1:7-13, 1973.

${ }^{15}$ Seiner, J.M. \& Reethof, G., "On the distribution of source coherency in subsonic jets", AIAA paper 74-4.

${ }^{16}$ Armstrong, R.R., Michalke, A. \& Fuchs, H.V., "Coherent structures in jet turbulence and noise", AIAA J., Vol. 15(7), pp. 1011-1017, 1977.

${ }^{17}$ Fuchs, H.V., "Space correlations of the fluctuating pressure in subsonic turbulent jets", J. Sound and Vibration, Vol. 23, pp. 77-99, 1972.

${ }^{18}$ Michalke, A., "On the effect of spatial source coherence on the radiation of jet noise", J. Sound and Vibration, Vol. 55(3), pp. 377-394, 1977.

${ }^{19}$ Panda, J., Seashotlz, R.G. and Elam, K.A., "Measurement of correlation between flow density, velocity and density*velocity2 with far field noise in high speed jets", 8th AIAA/CEAS Aeroacoustics Coference, June 17-19, Breckenridge, CO, AIAA 2002-2485, 2002.

${ }^{20}$ Bridges, J.E. \& Wernet, M.P. "Turbulence measurements of separate flow nozzles with mixing enhancement features", 8th AIAA/CEAS Aeroacoustics Conference and Exhibit, June 1719, Breckenridge, CO, 2002, AIAA Paper 2002-2484.

${ }^{21}$ Hileman, J. \& Samimy, M., "Turbulence structures and the acoustic far-field of a Mach 1.3 jet", AIAA J., Vol. 39(9), pp. 1716-1727, 2001.

${ }^{22}$ Hileman, J., Thurow, B. \& Samimy, M., "Acoustic source localization using a 3-D microphones array in a Mach 1.3 jet", 40th Aerospace Sciences Meeting \& Exhibit, January 14-17, Reno, NV, AIAA 2002-0366, 2002.

${ }^{23}$ Simonich, J. C., Narayanan, S., Barber, T. J. \& Nishimura, N., "Aeroacoustic characterization, noise reduction, and dimensional scaling effects of high subsonic jets," AIAA J., Vol. 39(11), pp. 2062-2069, 2001. 
AIAA Paper 2003-3314

${ }^{24}$ Narayanan, S., Barber, T.J. \& Polak, D.R., "High subsonic jet experiments: turbulence and noise generation studies", AIAA J., Vol. 40(2), pp. 430-437, 2002

${ }^{25}$ Lush, P.A., "Measurements of subsonic jet noise and comparison with theory," J. Fluid Mech. Vol. 46(3). pp. 477-500, 1971.

${ }^{26}$ Hussein, H.J., Capp, S.P., and George, W.K., "Velocity measurements in a high-Reynolds-number, momentumconserving, axisymmetric, turbulent jet," J. Fluid Mech. Vol. 258, pp. 31-75, 1994.

${ }^{27}$ Panchapakesan, N.R. and Lumley, J.L., "Turbulence measurements in axisymmetric jets of air and helium. Part 1: Air jets," J. Fluid. Mech. Vol. 246, pp. 197-223, 1993

${ }^{28}$ Stromberg, J.L. , McLaughin, D.K. and Troutt, T.R., "Flow field and acoustic properties of a Mach number 0.9 jet at a low Reynolds number," J. Sound Vib. Vol. 72(2), pp. 159176,1980 